\title{
Aerogelation of Polymer-Coated Photoluminescent, Plasmonic, and Magnetic Nanoparticles for Biosensing Applications
}

Laura Altenschmidt, "Sara Sánchez-Paradinas, "Franziska Lübkemann, Dániel Zámbó, Abuelmagd M. Abdelmonem, Henrik Bradtmüller, Atif Masood, Irene Morales, Patricia de la Presa, Alexander Knebel, Miguel Angel García García-Tuñón, Beatriz Pelaz, Karen D. J. Hindricks, Peter Behrens, Wolfgang J. Parak, and Nadja C. Bigall*

Cite This: ACS Appl. Nano Mater. 2021, 4, 6678-6688

Supporting Information

ABSTRACT: Macroscopic materials with nanoscopic properties have recently been synthesized by self-assembling defined nanoparticles to form self-supported networks, so-called aerogels. Motivated by the promising properties of this class of materials, the search for versatile routes toward the controlled assembly of presynthesized nanoparticles into such ultralight macroscopic materials has become a great interest. Overcoating procedures of colloidal nanoparticles with polymers offer versatile means to produce aerogels from nanoparticles, regardless of their size, shape, or properties while retaining their original characteristics. Herein, we report on the surface modification and assembly of various

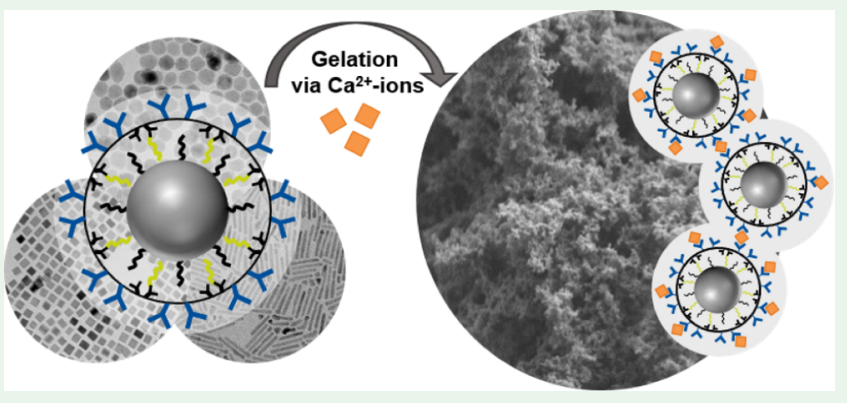
building blocks: photoluminescent nanorods, magnetic nanospheres, and plasmonic nanocubes with particle sizes between 5 and 40 $\mathrm{nm}$. The polymer employed for the coating was poly(isobutylene-alt-maleic anhydride) modified with 1-dodecylamine side chains. The amphiphilic character of the polymer facilitates the stability of the nanocrystals in aqueous media. Hydrogels are prepared via triggering the colloidally stable solutions, with aqueous cations acting as linkers between the functional groups of the polymer shell. Upon supercritical drying, the hydrogels are successfully converted into macroscopic aerogels with highly porous, open structure. Due to the noninvasive preparation method, the nanoscopic properties of the building blocks are retained in the monolithic aerogels, leading to the powerful transfer of these properties to the macroscale. The open pore system, the universality of the polymer-coating strategy, and the large accessibility of the network make these gel structures promising biosensing platforms. Functionalizing the polymer shell with biomolecules opens up the possibility to utilize the nanoscopic properties of the building blocks in fluorescent probing, magnetoresistive sensing, and plasmonic-driven thermal sensing.

KEYWORDS: nanoparticles, aerogels, polymer coating, phase transfer, versatile synthesis method

\section{INTRODUCTION}

Nanocrystal aerogels, as three-dimensional (3D) networks built from tailored nanoparticles, have recently been discovered as a new class of materials linking the nanoscopic to the macroscopic world, since building blocks forming ultralight macroscopic materials frequently exhibit still nanoscopic or even additional physical properties. ${ }^{1-5}$ Consequently, the development of further versatile routes toward the controlled assembly of presynthesized nanoparticles into such ultralight macroscopic architectures has been in the research focus of the recent past. $^{6-13}$ Synthetic pathways of nanoparticle aerogelation usually require first a hydrogelation step triggered by a controlled destabilization of the originally stable nanoparticle solution, which can be achieved by the partial removal of the surface ligands, by changing the polarity, by desalting, by the addition of divalent or trivalent cations, by coordination chemistry, and many more, ${ }^{6,8,14,15}$ always with the necessity of precise tuning of the chemical route for the respective nanoparticle system. After hydrogelation, supercritical drying is a common method to transfer the macroscopic nanoparticle assembly from liquid to air environment. Alternatively, the so-called cryaerogelation procedure can be employed for synthesizing aerogels, in which the assembly is triggered by ice crystallite templates followed by the freeze drying of the formed assemblies. ${ }^{9,16-18}$ It is important to note that except for few examples on lyogelating nanoparticles from inorganic solvents, ${ }^{8}$ the nanoparticles first need to be

Received: $\quad$ March 4, 2021

Accepted: May 26, 2021

Published: July 8, 2021 
Scheme 1. Schematics of the Aerogel Preparation Procedure ${ }^{a}$

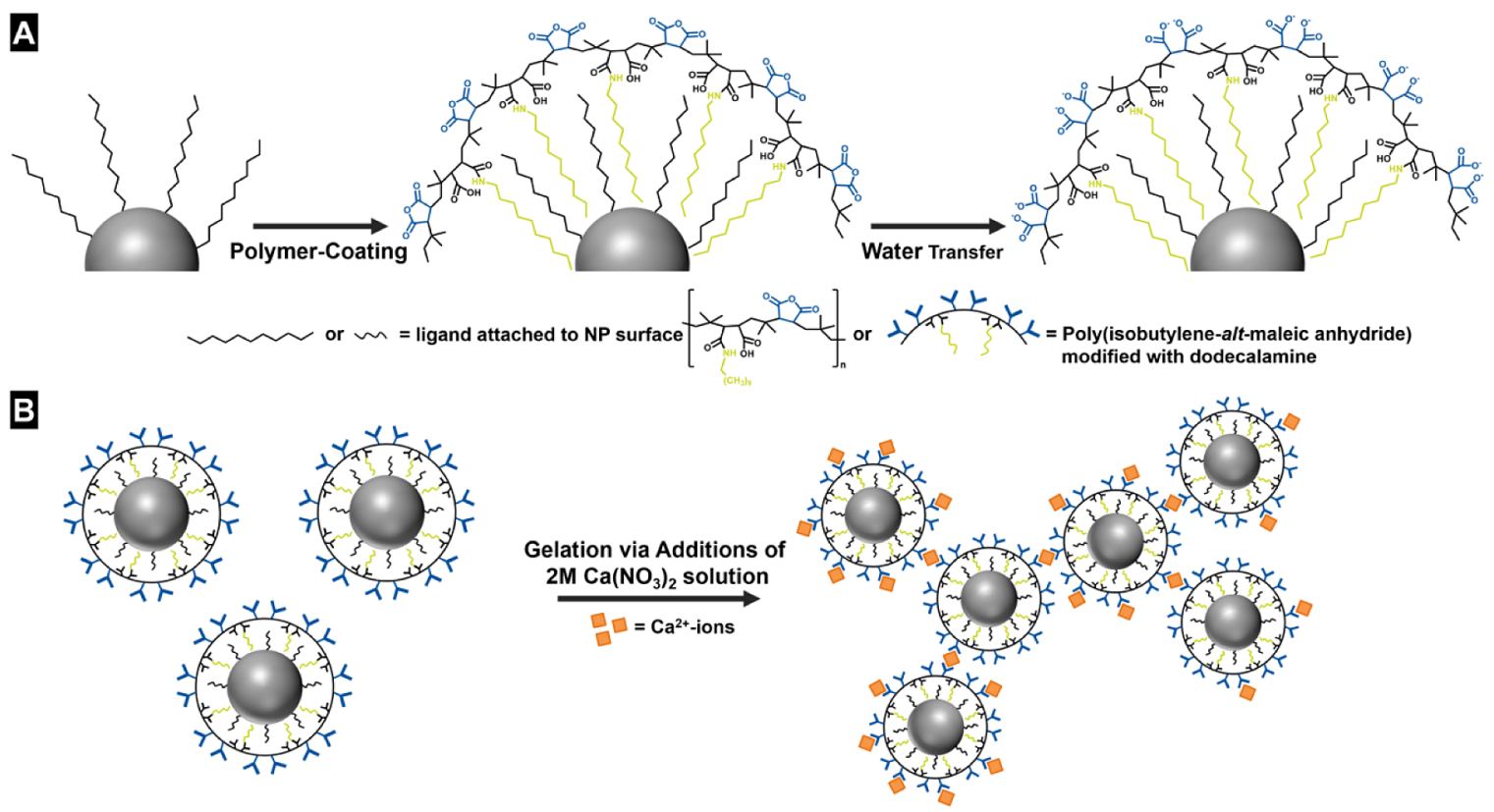

a(A) The nanoparticles are first coated with the amphiphilic polymer, which ensures their stability in aqueous solutions. (B) In the second stage, the self-assembly of the aqueous nanoparticle solutions is triggered via $\mathrm{Ca}^{2+}$ ions facilitating the formation of gel structures (hydrogels). Aerogels are prepared from the corresponding hydrogels by means of supercritical drying.

transferred into polar solutions before the gelation can be initiated.

Synthesis of the building blocks-nanoparticles in various shapes, sizes, and compositions-has gone through a steep development in the past years. Photoluminescent, magnetic, and plasmonic nanoparticles are of special interest for applications due to their size-, shape-, and surface chemistrydriven properties. Importantly, the combination of the abovementioned properties in a single material is of high interest from applicational point of view. ${ }^{19}$ So far, these nanoparticles were already successfully applied in various fields such as in the development of nanomedicines, ${ }^{20,21}$ as contrast agents in magnetic resonance imaging, ${ }^{22}$ or for general imaging purposes, ${ }^{23}$ as well as in optical sensing. ${ }^{24}$ However, the synthesis of novel, high-quality nanoparticles is performed in organic solvents, and thus, the hydrophobic ligands hinder the formation of hydrogels right after the synthesis. Consequently, the nanoparticles need to be transferred from organic to aqueous media under retention of the nanoscopic properties as well as their stability. ${ }^{25,26}$ Multiple different methods have already been established for the exchange of surface ligands or encapsulation in amphiphilic polymer shells. ${ }^{27}$ While ligand exchange frequently leads to irreversible changes at the nanoparticle surface itself (formation of vacancies, surface traps, etc.), the preservation of the initial nanoscopic properties cannot be ensured. Additionally, these methods must be optimized for each nanoparticle systems due to the lack of a universal exchange strategy.

A promising solution to this issue is an alternative phase transfer route, in which no ligand exchange is necessary to be carried out. ${ }^{28-31}$ Here, an amphiphilic polymer (consisting of a hydrophilic backbone and hydrophobic side chains) is wrapped around the nanoparticles in a way that the side chains can intercalate or surround the hydrophobic nanoparticle ligands, and the backbone provides solubility in aqueous media. This method has received a lot of interest in research in the recent past because it is highly versatile due to its nonspecific nature. Via merging the versatility and tunability of the polymer coating with the porosity and accessibility of the aerogel structures, new routes can be opened toward applications.

Polymer-coated nanoparticles can be used as versatile biosensing units depending on the properties of the core nanoparticles and the functionalization of the polymer shell. Photoluminescent nanoparticles are promising Förster resonance energy transfer (FRET) units allowing the enhancement of the detected signal in nanobiosensing upon incorporating the acceptor molecules directly into the amphiphilic polymer. ${ }^{32}$ Using plasmonic core nanoparticle, the plasmonic-driven thermal effect can be utilized for sensing cancer markers. ${ }^{33}$ Superparamagnetic nanoparticles are widely used in bioimaging and biomedical detection due to their relatively low cost, ease of functionalization, and biocompatibility. ${ }^{34}$ Amphiphilic polymers around the magnetic nanoparticle cores offer a universal approach to extend the functionalization possibilities of the core-shell nanoparticles with biomolecules, which make these systems magnetothermally resistive sensing units. ${ }^{35}$ Importantly, assembling these sensing units into gel structures further expand the application potential of these systems. Nondestructive gelation methods ensure the formation of a homogeneous, 3D structure with high porosity and open pore system. Aerogelating of these hyperbranched backbones opens paramount possibilities toward the sensing of gaseous analytes as well, which cannot be achieved in solely dried nanoparticle solutions.

In this work, we show that polymer-coated nanocrystals can be successfully used as building blocks in aerogel fabrication, which lead to macroscopic aerogel monoliths with retained nanoscopic properties (Scheme 1). The advantage of polymer coating prior to gelation is that the polymer-coating procedure 
is applicable to nearly all kinds of nanoparticles from nonpolar syntheses so that the established route is highly versatile for assembling various types of nanoparticle assemblies into ultralight composites. To show this key advantage, three examples are demonstrated: photoluminescent $\mathrm{CdSe} / \mathrm{CdS}$, superparamagnetic iron-oxide (iron oxide nanoparticles (IONs)), and plasmonic $\mathrm{Au}_{3} \mathrm{Cu}$ nanoparticles-all of them synthesized by high-boiling state-of-the-art synthesis but differing in size and shape (being rod-shaped, quasi-spherical, and cubic, respectively). These nanoparticles are transferred by this route into aqueous solution using poly(isobutylene-altmaleic anhydride) (PMA) modified with 1-dodecylamine side chains (75\% 1-dodecylamine molecules with respect to the number of PMA monomers), following the route of Lin et al. ${ }^{36}$ The formation of hydrogels are triggered via the addition of $\mathrm{Ca}^{2+}$ cations. To further increase the accessibility of the polymer-coated nanoparticle gel networks, formation of aerogel monoliths is performed by supercritical drying. By providing a novel gelation route for polymer-encapsulated nanoparticles, a universal pathway for the synthesis of ultralight materials composed of self-assembled high-quality nanoparticles is developed. The noninvasive polymer coating as well as the subsequent mild gelation route of the nanoparticles ensure that the nanoscopic optical and magnetic properties can be preserved in the macroscopic assemblies. The key advantage of the presented procedure is the opportunity to exploit the special properties of the building blocks (being optically active or magnetically responsive) and of the polymer (being modifiable by biomolecules). The 3D structure and the open pore system of the studied aerogels allow their utilization in biosensing applications, with special interest in fluorescence probing, magnetoresistive sensing, and plasmonic-driven thermal sensing.

\section{EXPERIMENTAL SECTION}

Synthesis of Rod-Shaped CdSe/CdS Nanoparticles. In the first stage, CdSe quantum dots (seeds) were prepared following the procedure of Carbone et al. ${ }^{37}$ with minor modifications. Typically, trin-octylphosphine oxide (TOPO, $3.0 \mathrm{~g}$ ), octadecylphosphonic acid $(\mathrm{ODPA}, 0.280 \mathrm{~g})$, and CdO $(0.060 \mathrm{~g})$ were mixed in a $50 \mathrm{~mL}$ flask, heated to $150{ }^{\circ} \mathrm{C}$, and degassed under vacuum for $1 \mathrm{~h}$. Then, under argon, the solution was heated to $300{ }^{\circ} \mathrm{C}$ to dissolve the $\mathrm{CdO}$ until a clear and colorless solution was obtained. At this point, $1.8 \mathrm{~mL}$ of tri$n$-octylphosphine (TOP) was injected into the flask, and the temperature was allowed to rise to $380{ }^{\circ} \mathrm{C}$. Once at this temperature, a $1: 1 \mathrm{Se} / \mathrm{TOP}$ solution $(0.058 \mathrm{~g} \mathrm{Se}+1.8 \mathrm{~mL}$ TOP) was quickly injected. The temperature was allowed to recover before cooling the flask. By this route, red fluorescent CdSe seeds were synthesized. After the synthesis, the nanoparticles were precipitated with methanol, washed by repeated dispersing and redispersing in toluene, and precipitated by the addition of methanol, and they were finally dissolved and stored in toluene.

In a second step, $\mathrm{CdSe} / \mathrm{CdS}$ nanorods (NRs) were synthesized by a seeded-growth approach based on the protocol reported by Carbone et al. ${ }^{37}$ The synthesis is based on the anisotropic growth of CdS over previously prepared spherical CdSe seeds. Briefly, CdO was first degassed and then decomposed in the presence of TOPO. Typically, CdO $(0.060 \mathrm{~g})$ was mixed in a flask with TOPO $(3.0 \mathrm{~g})$, ODPA $(0.280 \mathrm{~g})$, and hexylphosphonic acid $(0.080 \mathrm{~g})$. The flask was first degassed under vacuum for about $1 \mathrm{~h}$ at $150{ }^{\circ} \mathrm{C}$ and then heated up to $300{ }^{\circ} \mathrm{C}$ under argon. Once at that temperature, $1.8 \mathrm{~mL}$ of TOP was injected, and the resulting solution was heated to $380^{\circ} \mathrm{C}$ under argon. At this point, a mixture of TOP solution containing the sulfur precursor $(0.130 \mathrm{~g} \mathrm{~S}+1.8 \mathrm{~mL} \mathrm{TOP})$ and the seeds in toluene (the concentration of CdSe quantum dots serving as seed particles in the toluene solution was always $400 \mu \mathrm{M})$ were quickly injected. The concentration has been calculated by measuring the absorption spectrum and determining the diameter and the extinction coefficient of the $\mathrm{CdSe}$ seeds using the equations given by $\mathrm{Yu}$ et al. ${ }^{38}$ After injection, the temperature dropped to $270-300{ }^{\circ} \mathrm{C}$, and the solution was left to recover the temperature for 8 minutes before removing the heating mantle. The obtained NRs were washed by repeated precipitation with $\mathrm{MeOH}$ for several times followed by re-dissolution and finally stored in chloroform.

Synthesis of Quasi-Spherical IONs. IONs were synthesized following the procedure of $\mathrm{Yu}$ et al., ${ }^{39}$ with minor modifications. In a typical synthesis, $0.178 \mathrm{~g}$ of $\mathrm{FeO}(\mathrm{OH})$ and $3.05 \mathrm{~g}$ of oleic acid were mixed with $5.55 \mathrm{~g}$ of 1 -octadecene (ODE) in a $25 \mathrm{~mL}$ three-neck round flask. The mixture was heated to $320{ }^{\circ} \mathrm{C}$ under argon and kept at this temperature for $1 \mathrm{~h}$. During the heating, the iron source dissolved, and the solution turned from colorless to light brown and finally to dark brown. The nanoparticles were purified by precipitation with $\mathrm{MeOH}$ and redissolution in toluene twice. The resulting nanoparticles were finally stored in chloroform.

Synthesis of Cubic $\mathrm{Au}_{3} \mathrm{Cu}$ Bimetallic Nanoparticles. $\mathrm{Au}_{3} \mathrm{Cu}$ nanocubes (NCs) were synthesized following a previously reported procedure ${ }^{40}$ with some modifications. Typically, for the synthesis of 5-6 nm cubes, a mixture of $\mathrm{HAuCl}_{4}(100 \mathrm{mg}), \mathrm{Cu}(\mathrm{acac})_{2}(65 \mathrm{mg}), 1-$ adamantanecarboxylic acid (ACA, $270 \mathrm{mg}$ ), 1,2-hexadecanediol (HDD, $1.6 \mathrm{~g})$, 1-hexadecylamine (HAD, $2 \mathrm{~g}$ ), diphenylether (DPE, $10 \mathrm{~mL}$ ), and 1-dodecanthiol (DDT, $500 \mu \mathrm{L}$ ) were mixed together in a $25 \mathrm{~mL}$ three-neck flask. The reaction mixture was heated to $180{ }^{\circ} \mathrm{C}$ at a rate of $8{ }^{\circ} \mathrm{C} \mathrm{min}{ }^{-1}$ and held at $180{ }^{\circ} \mathrm{C}$ for $20 \mathrm{~min}$. After this time, the solution exhibits a dark blue color. The solution was centrifuged twice with chloroform and finally redissolved in chloroform.

Synthesis of the Amphiphilic Polymer. The polymer was synthesized by the procedure of Lin et al. ${ }^{24}$ The precursor polymer PMA (3.084 g, $20 \mathrm{mmol}$ monomer) was dispersed in $80 \mathrm{~mL}$ anhydrous tetrahydrofuran (THF). 1-Dodecylamine ( $2.78 \mathrm{~g}, 5 \mathrm{mmol}$; $75 \%$ with respect to the monomer units) solubilized in $20 \mathrm{~mL}$ anhydrous THF was added to the polymer dispersion under vigorous stirring at $60{ }^{\circ} \mathrm{C}$. Within the first few minutes, the solution became clear due to the reaction of the amine with the anhydride. After $3 \mathrm{~h}$, the solution was concentrated in a rotary evaporator system at $p=300$ mbar to roughly a third of the initial volume. The concentrated solution was further incubated at $60^{\circ} \mathrm{C}$ for at least $3 \mathrm{~h}$ under stirring. Finally, THF was completely removed, and the resulting slightly yellow solid was redispersed in $40 \mathrm{~mL}$ anhydrous chloroform, yielding a polymer concentration of $0.5 \mathrm{M}$ with respect to the monomer units.

Water Transfer of Nanoparticles. The respective amount of polymer (see Table 1) was added to the nanoparticles in chloroform, and the mixture was stirred between $30 \mathrm{~min}$ and hours $(30 \mathrm{~min}$ for $\mathrm{NCs}$ and NRs; $1.5 \mathrm{~h}$ for IONs).

Table 1. Polymer-Coating Conditions for the Different Types of Nanoparticles and the Corresponding Amount of Monomer

\begin{tabular}{lcccc}
$\begin{array}{c}\text { nanoparticle } \\
\text { type }\end{array}$ & $\begin{array}{c}\text { nanoparticle } \\
\text { concentration } \\
(\mathrm{M})\end{array}$ & $\begin{array}{c}\text { volume } \\
\text { nanoparticle } \\
(\mu \mathrm{L})\end{array}$ & $\begin{array}{c}\text { volume } \\
\text { polymer } \\
(\mathrm{mL})\end{array}$ & $\begin{array}{c}\text { monomer (per } \\
\text { nanoparticle/ } \\
\left.\text { per } \mathrm{nm}^{2}\right)\end{array}$ \\
nanorods & 0.14 & 500 & 5 & $36 / 0.06$ \\
nanocubes & 0.019 & 500 & 2 & $105 / 0.58$ \\
IONs & 0.24 & 100 & 5 & $104 / 0.11$ \\
\hline
\end{tabular}

Afterward, the solvent was completely removed on a rotavapor system under reduced pressure, and the precipitate was redispersed in $0.1 \mathrm{M} \mathrm{NaOH}$. The aqueous phase was then filtered through a hydrophilic syringe filter (Millipore Millex-GP PES $0.22 \mu \mathrm{m}$ pore size), and the alkaline solution was slowly exchanged by deionized water via centrifugation $(\mathrm{rcf}=3773)$ in a centrifuge filter (molecular weight cutoff, $\mathrm{MWCO}=30$ 000). The filtered solvent was discarded, and the residue was redispersed in deionized water. The procedure was repeated five times on average. The successful polymer coating and water transfer have been tested by performing gel electrophoresis 

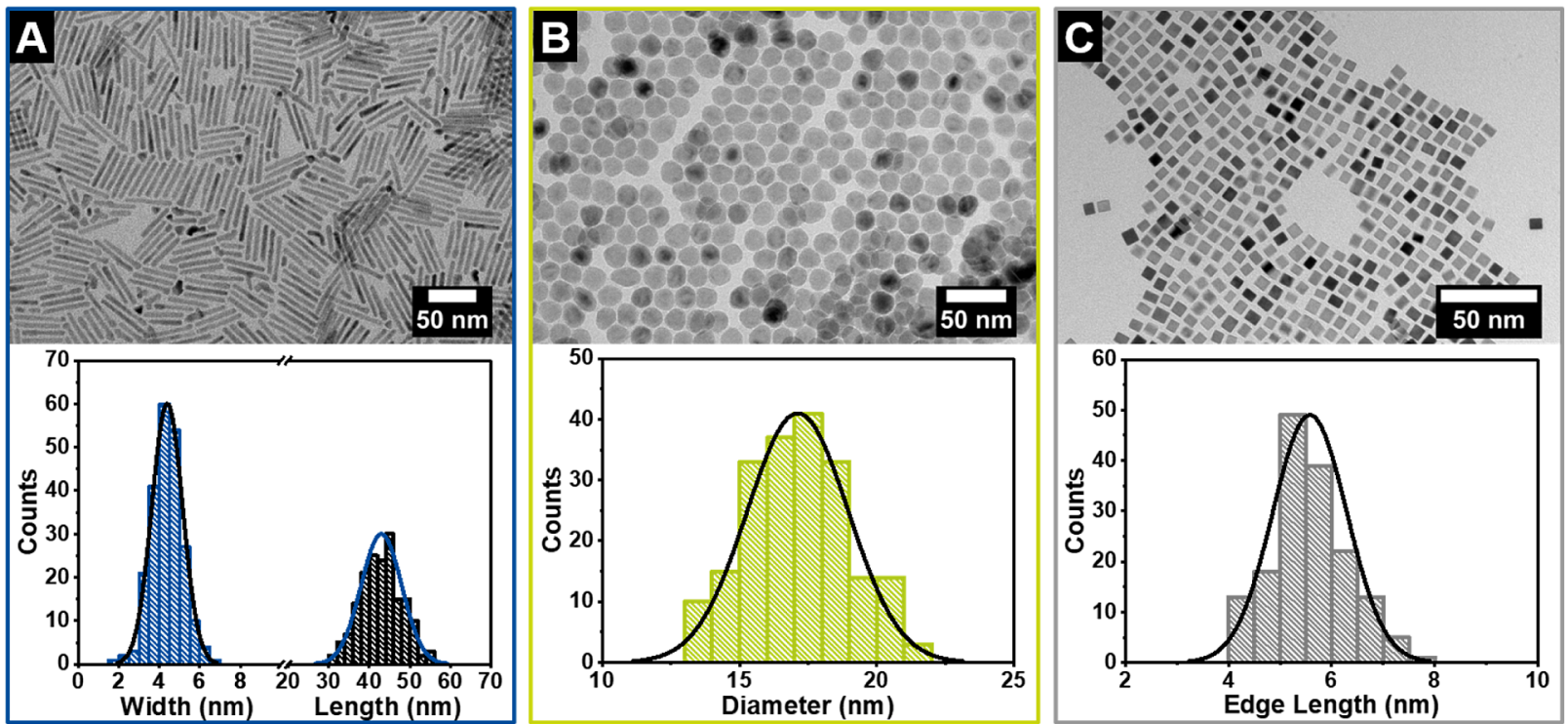

Figure 1. TEM images and corresponding size distributions of the as-synthesized nanoparticles in organic solution. (A) dot-in-rod-shaped CdSe/ CdS NRs with an average length of $43 \pm 5 \mathrm{~nm}$ and average width of $4 \pm 1 \mathrm{~nm}$; (B) spherical IONs with an average diameter of $17 \pm 2 \mathrm{~nm}$; and (C) $\mathrm{Au}_{3} \mathrm{Cu}$ NCs with an average edge length of $6 \pm 1 \mathrm{~nm}$.

for $1 \mathrm{~h}$ at $110 \mathrm{~V}$ and $50 \mathrm{~mA}$ in an agarose gel $(1.5 \%$ agarose in $0.5 \times$ Tris-borate-EDTA (TBE) buffer) with $0.5 \times \mathrm{TBE}$ as the running buffer. The procedure was successful when a narrow band ran through the gel. Finally, the water-transferred nanoparticles were cleaned from the excess of polymer micelles by ultracentrifugation at $80000 \mathrm{~g}$ twice for $1 \mathrm{~h}$ each and washing them with deionized water. Finally, the precipitate was redispersed in deionized water.

Gel Preparation. Hydrogels were prepared by adding a $2 \mathrm{M}$ $\mathrm{Ca}\left(\mathrm{NO}_{3}\right)_{2}$ solution to the cleaned polymer-coated nanoparticles (the volume of the nanoparticle solution was always adjusted with deionized water to $1 \mathrm{~mL}$ ). The mixture was shortly vortexed and left in a vibration-free area for several minutes or hours, depending on the material. The average time for complete gelation was within 15 min for NRs, ranging from $45 \mathrm{~min}$ to $1 \mathrm{~h}$ for NCs, and up to several hours for IONs, and this is probably caused by the low particle concentration. The obtained gels were washed with water $(1 \mathrm{~mL})$ five times on average after completing the gelation time to obtain the hydrogels.

For the preparation of aerogels, the solvent was exchanged from water to dry acetone at ambient conditions: First, the water was partially removed and slowly replaced with acetone, followed by the partial removal of this new water and acetone mix and by the replacement with fresh acetone. This process was repeated with the resulting mixture until the entire solution has been replaced with acetone. Finally, the dried acetone was introduced in the same manner to entirely remove any residual water. The samples were placed into a supercritical dryer (E3100 from Quorum Technologies), whereby the dry acetone was first exchanged with liquid $\mathrm{CO}_{2}$ and kept overnight. Then, the supercritical dryer was heated to $31.1{ }^{\circ} \mathrm{C}$, causing the transition from the liquid phase to the supercritical phase (at 73.8 bar). Finally, the gaseous $\mathrm{CO}_{2}$ was slowly removed from the chamber, and the aerogels were obtained.

Instrumentation. The optical characterization of the nanoparticle solutions and gels was performed at room temperature as follows.

PL Spectra and Quantum Yields. The photoluminescence (PL) spectra (of all samples, that is, aerogels, hydrogels, and all nanoparticles in solution) were recorded by means of a dual-FL spectrofluorometer (Horiba Scientific) equipped with a Quanta- $\varphi$ integrating sphere. Photoluminescence quantum yields (PLQY) were determined by measuring in absolute mode, that is, comparing the number of emitted photons with the number of absorbed photons. The latter is the intensity decrease in the incident (excitation) light intensity caused by the sample. Therefore, corrections regarding reflection losses and the characteristics of the empty integration sphere were also performed.

Fluorescence Lifetime. Time-correlated single photon counting (TCSPC, fluorescence lifetime) measurements were performed on a Fluoromax-4 spectrometer combined with the Fluorohub TCSPC unit, using two-pulsed diodes (operating at 368 and $454 \mathrm{~nm}$, respectively) with a pulse full width at half-maximum (FWHM) of less than $1.6 \mathrm{~ns}$ and a repetition rate of $500 \mathrm{kHz}$.

Extinction/Absorption. The extinction spectra of the colloidal solutions before and after polymer coating were recorded in transmission mode using a $3 \mathrm{~mL}$ quartz cuvette in a Cary 5000 absorption spectrometer because the nanoparticle solutions were clear and not cloudy, pointing toward negligible scattering effects. For measuring hydrogels, due to their highly scattering nature, absorption measurements were carried out by means of an integration sphere. Therefore, the hydrogels were directly prepared inside a $3 \mathrm{~mL}$ quartz cuvette. The beam was focused directly onto the gel by refocusing the mirrors, and the absorption was measured from 300 to $800 \mathrm{~nm}$ in a Cary 5000 diffuse reflectance accessory. For the same reasons, absorption spectra of the aerogels were measured using a solid sample holder inside the Quanta- $\Phi$ sphere in a dual-FL instrument by Horiba in IN and OUT positions. The absorption of the samples was calculated from the 3D plot by MatLab, using the logarithmic ratio of the light intensity when the sample is diffusely illuminated by the integrating sphere's surface to the emission intensity when the sample is directly excited by the incident beam. The absorption of the empty sample holder was subtracted.

Microscopic Measurements. Transmission electron microscopy (TEM) imaging was carried out on a FEI-Tecnai G2 F20 microscope, operating at a $200 \mathrm{kV}$ accelerating voltage. Scanning TEM high-angle annular dark-field (STEM-HAADF) and energy-dispersive X-ray spectroscopy (EDX) measurements were carried out with a Jeol JEM-2100F microscope equipped with a field emission gun operated at $200 \mathrm{kV}$. The samples for TEM measurements were prepared by drop-casting diluted solutions of the colloids or hydrogel fragments onto copper grids (QUANTIFOIL), with subsequent evaporation of the solvent. Aerogels were deposited on the TEM grids by pressing them slightly on the aerogel samples. Scanning electron microscopy (SEM) was carried out with a JEOL JSM 6700F field emission scanning electron microscope. The samples for SEM imaging were prepared by deposition of small pieces of the hydrogels on carbon holders and of the aerogels on adhesive carbon films. It should be noted that the hydrogel, after drying on the TEM grid and the carbon 

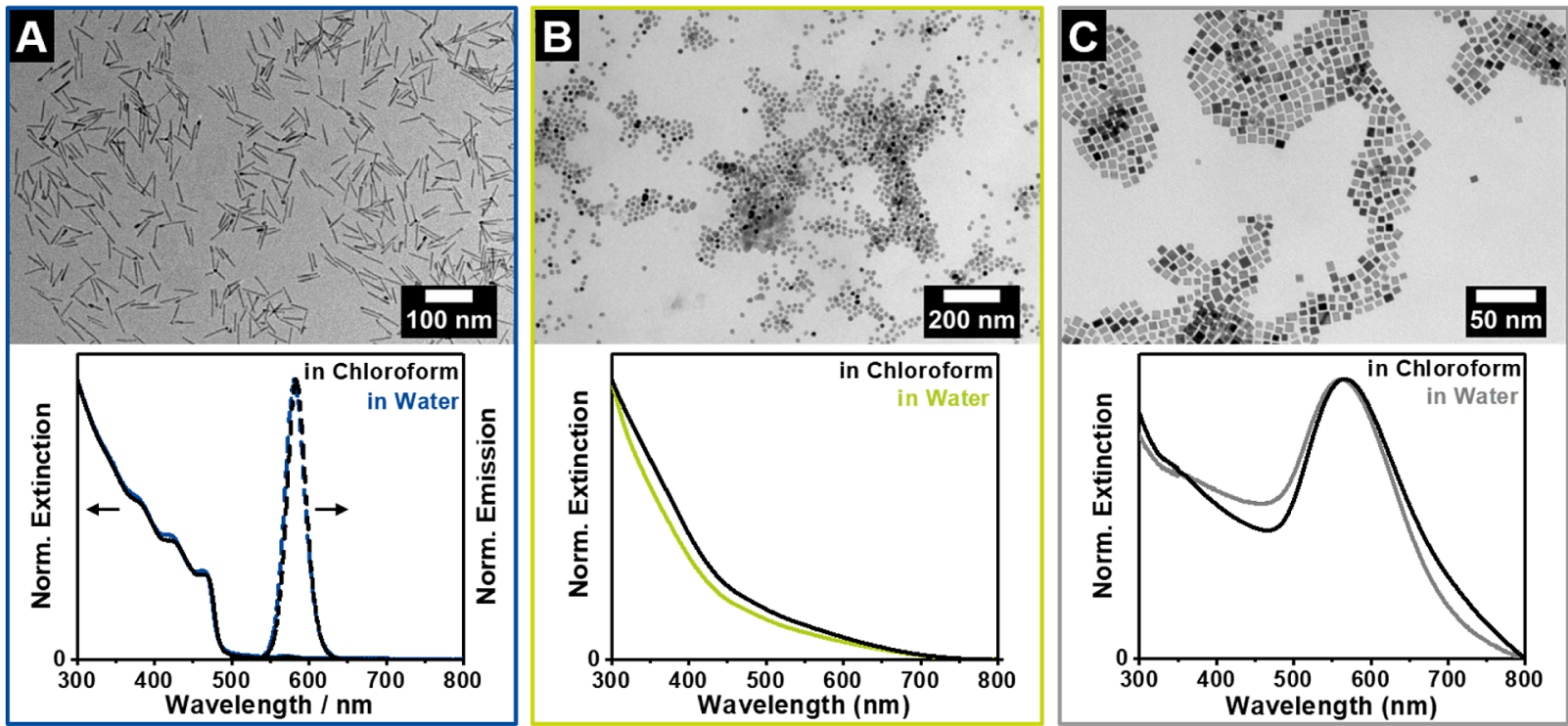

Figure 2. TEM images and normalized extinction and emission spectra of polymer-coated nanoparticles in organic and aqueous solution. (A) TEM image of dot-in-rod-shaped CdSe/CdS NRs in aqueous solution, and normalized extinction (solid line) and emission spectra (dashed line) of NRs in organic solution (black lines) and aqueous solution (blue lines). (B) TEM image of spherical IONs in aqueous solution and normalized extinction spectra of IONs in organic (black line) and aqueous solution (green line). (C) TEM image of $\mathrm{Au}_{3} \mathrm{Cu}$ NCs in aqueous solution and normalized extinction spectra of NCs in organic solution (black line) and aqueous solution (gray line).

holder, will not be a hydrogel but a xerogel since it collapses due to the occurrence of capillary forces during the drying.

Magnetic Measurements. The magnetic characterization was performed in a SQUID (superconducting quantum interference device) magnetometer Quantum Design MPMS-5S. For the measurement of the uncoated IONs in chloroform suspension and IONs with polymer shell, $50 \mu \mathrm{L}$ of the suspension was dropped in a piece of cotton. Zero field cooled (ZFC) magnetization curves were measured with a maximum applied field of $50 \mathrm{kOe}$ at 5 and $300 \mathrm{~K}$. Besides, $M$ versus $H$ curves were measured under field cooled (FC) procedure under different cooling fields $H_{\text {cool }}=5,10$, and $30 \mathrm{kOe}$. ZFC and FC thermal magnetization curves were measured from 10 to $300 \mathrm{~K}$ at different cooling fields: $H_{\text {cool }}=0.1,1,2$, and 5 kOe. A similar methodology was followed for the IONs in aerogels.

Argon Physisorption Measurements. Argon physisorption isotherms were carried using a Micromeritics 3Flex instrument at $87 \mathrm{~K}$. Prior to the measurements, the samples were degassed under vacuum at $25{ }^{\circ} \mathrm{C}$, for $24 \mathrm{~h}$. Brunauer-Emmet-Teller (BET) equation was applied to determine the specific surface areas. All evaluations were performed via 3Flex Software Version 5.02.

\section{RESULTS AND DISCUSSION}

Nanoparticle Characterization. Figure 1 shows TEM images of the different types of as-synthesized rod-shaped semiconductor, quasi-spherical magnetic nanoparticles, and plasmonic cubic nanoparticles. In all these cases, a narrow size distribution and a homogeneous morphology could be obtained: the CdSe/CdS NRs were $43 \pm 5 \mathrm{~nm}$ in length and had an average width of $4 \pm 1 \mathrm{~nm}$, IONs exhibited a diameter of $17 \pm 2 \mathrm{~nm}$, and the $\mathrm{Au}_{3} \mathrm{Cu}$ NCs had an average edge length of $6 \pm 1 \mathrm{~nm}$.

Polymer Coating of the Building Blocks. Nanoparticles were coated by means of an amphihilic polymer to transfer them from organic solution to aqueous solution. ${ }^{36}$ To date, ligand exchange on the organic nanoparticles has been employed as a common strategy before transferring the nanoparticles to aqueous media prior to the gelation. ${ }^{26}$ The phase transfer via ligand exchange frequently results in a deterioration of the original nanoscopic properties (such as loss of fluorescence $\mathrm{QY}$ ), and sometimes, in the partial loss of colloidal stability. There is solely one example of nanoparticle gelation directly from nonpolar solvents as reported by our group ${ }^{8}$ and an all-inorganic gelation route by the Eychmüller group. ${ }^{7}$ In our recent strategy, hydrazine was employed as a destabilizing agent. However, even though this destabilization route was observed to be applicable for various types of nanoparticles, the resulting gels are more compact than those obtained by employing a previous phase transfer to aqueous solutions. Polymer coating was previously reported to be a suitable technique to transfer a huge variety of nanoparticles from organic to aqueous media, while largely retaining their original properties. ${ }^{28-30}$ This motivated us to use this water transfer route prior to the gelation of the nanoparticles. The extinction spectra (and emission in the case of the semiconductor nanoparticles) of the original nanoparticles and those after polymer coating are compared in Figure 2.

It can be seen that within the accuracy of our measurement setup, the optical spectra exhibit no significant changes after the polymer coating for all types of tested nanoparticles. For QY, a value of $32 \%$ could be obtained, which remained unchanged with lifetimes of 27 and 30 ns. For the case of NCs, the slight shift of the absorbance maximum can be attributed to the changed dielectric properties of the chemical environment as well as to a certain degree of instability of the cubes when the diameter is below $10 \mathrm{~nm}$, as described by Liu et al. ${ }^{40}$ Nevertheless, it can be concluded that the plasmonic properties are not affected by the polymer shell. For the nanospheres (NSs), only a shift of the absorbance spectrum toward higher wavelengths can be seen, but the trend of the curve remains unchanged.

The conclusions from the optical characterization are confirmed by the TEM analysis (see Figure 2, lower row) as well. In the images, no significant amounts of polymer can be seen (except for the NSs, which show negligible amount of residual polymer). Therefore, we can conclude that the excess polymer could be sufficiently removed from the solution by 

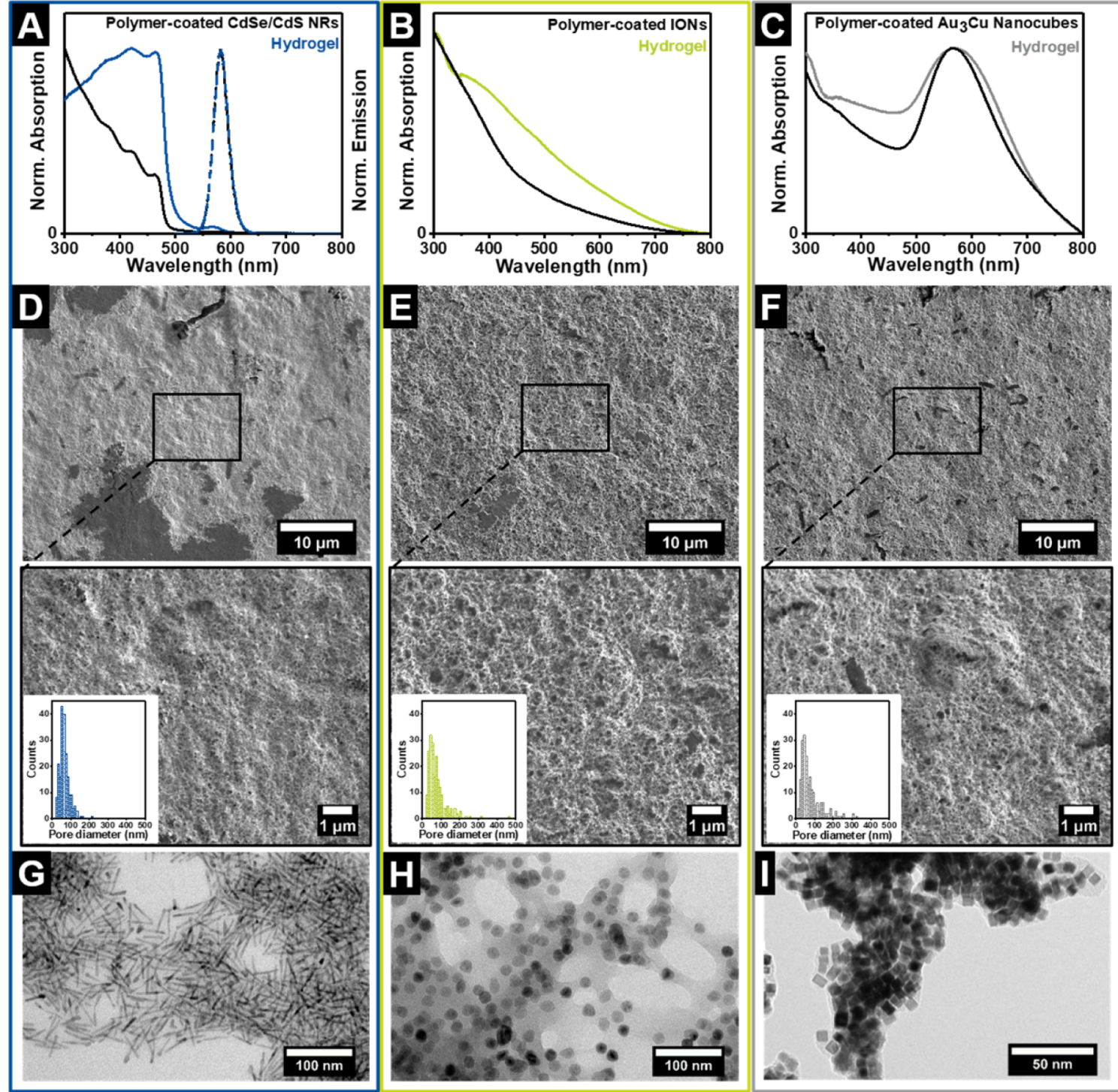

Figure 3. Optical and structural properties of the hydrogels. Normalized absorption spectra of the polymer-coated nanoparticles in aqueous solution and their corresponding hydrogels for (A) CdSe/CdS NRs, (B) spherical IONs, and (C) $\mathrm{Au}_{3} \mathrm{Cu}$ NCs. SEM images with lower and higher magnifications $(\mathrm{D}-\mathrm{F})$ and TEM images of the obtained xerogels $(\mathrm{G}-\mathrm{I})$ from $(\mathrm{D}$ and $\mathrm{G}) \mathrm{CdSe} / \mathrm{CdS} \mathrm{NRs},(\mathrm{E}$ and $\mathrm{H}) \mathrm{IONs}$, and $(\mathrm{F}$ and $\mathrm{I}) \mathrm{Au}_{3} \mathrm{Cu}$ NCs. The insets of the panels $\mathrm{D}-\mathrm{F}$ show the pore size distributions of the corresponding xerogels.

ultracentrifugation (following the route of del Pino and coworkers), ${ }^{41}$ yielding nanoparticles with a thin layer of polymer. ${ }^{42}$ The particle alignment observable from the transmission electron micrographs in Figure 1 (before coating) is different from that of Figure 2 (after the phase transfer). This can be explained by the increased surface charge of the polymer-coated nanoparticles and the concomitantly higher Coulomb and steric repulsion forces. Additionally, different interactions to the underlying TEM carbon substrate grid are expected. Nevertheless, by this route, no change in size or shape of the inorganic nanoparticles was observed (see Figure S1 for the DLS size distribution), as it previously was proven for different nanoparticles transferred by the polymer-coating procedure. ${ }^{28-30}$ Therefore, we conclude that in this work, the nanoparticles and their morphological and optical properties can be successfully preserved upon water transfer procedure.

Hydrogels. For the preparation of nanoparticle gel networks, aqueous cations were added to the polymer-coated particles. The use of cations to induce gelation was previously reported by Wen et $\mathrm{al}^{43}$ The authors used calcium ions as a destabilizing agent to prepare gels from citrate-coated Pd nanoparticles. In this work, the addition of divalent cations induces the assembly of the nanoparticles by interacting with the carboxylic acid moieties present in the polymer shell surrounding the nanoparticles. On the one hand, the interaction of the acid functionality with the cations reduces the surface charge of the polymer-coated nanoparticles and ultimately causes destabilization and gelation. On the other hand, $\mathrm{Ca}^{2+}$ ions can simultaneously bind to the $\mathrm{COO}^{-}$groups of two nanoparticles, facilitating their cross-linking. As a support for the latter explanation, during the optimization of the gelation procedure, it was found that only salts with divalent cations trigger the gelation. Therefore, $\mathrm{Ca}\left(\mathrm{NO}_{3}\right)_{2}$ was chosen for all the experiments. It has to be emphasized that since the destabilization process is based on the interaction between the cations and the carboxylic groups of the polymer, a part that is common in all the three systems used, the gelation process is universal and independent of the nature of 

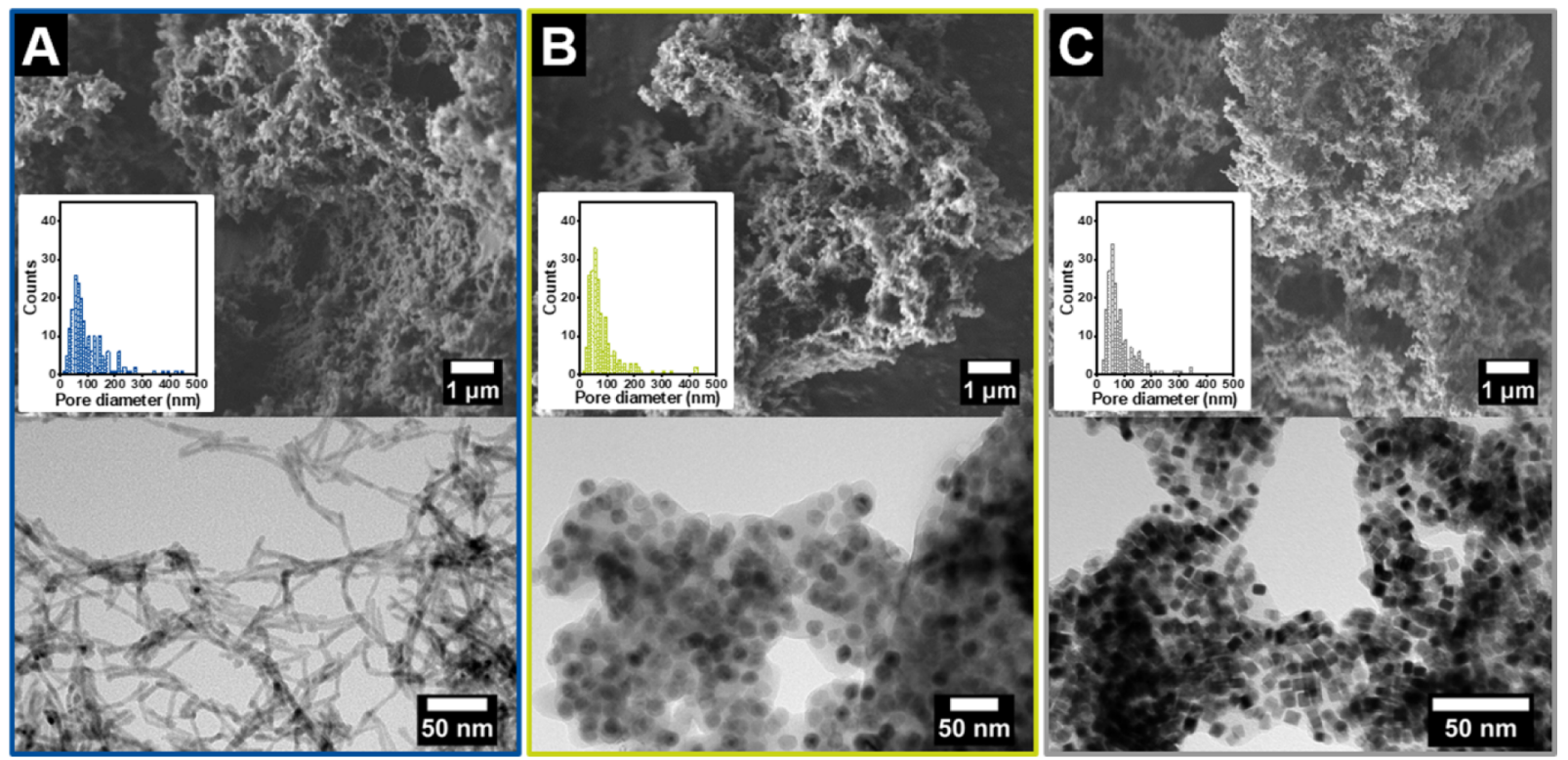

Figure 4. SEM images (upper row) and TEM images (bottom row) of the obtained aerogel monoliths of the (A) CdSe/CdS NRs, (B) spherical IONs, and (C) $\mathrm{Au}_{3} \mathrm{Cu}$ NCs. The insets show the pore size distributions of the corresponding aerogels.

the nanoparticle. For all systems, monolithic structures could be obtained by this method, which exhibited approximately only $20 \%$ volume shrinkage from initial solution to the hydrogel (see Figure S2 for photographs).

The obtained hydrogel monoliths were thoroughly characterized via their optical spectrum and electron microscopy (SEM and TEM) to verify the preservation of the previous measured nanoscopic properties after the gelation process. As shown in Figure 3A-C, the absorption spectra show slight deviations from the free nanoparticles in all types of gels, which can be explained by the interparticle interactions in the gel structure due to reduced distances. However, the typical characteristics are still retained. For NRs, a shift of the CdS absorption edge toward longer wavelengths can be seen, and the absorption goes into saturation after the absorbance edge, which is typical for gels due to the increased optical density of nanocrystal gels. ${ }^{4,15}$ This also manifests in a certain decrease in the QY (to $25.8 \%$ ), due to the reabsorption of the emitted light by the optically dense gel structure. The interparticle interactions cause a broadening of the plasmon peak for the NC hydrogels and a shift of the absorption toward higher wavelengths in the case of the NSs, but the trend of the curve remains the same. These observations show that the nanoscopic properties still exist in the hydrogel structure, even though they are slightly altered due to the increase of interactions between the polymer-coated nanoparticles.

The morphology of the dried hydrogels (that is, xerogels), analyzed by SEM (insets of Figure 3D-F), shows a continuous and homogeneous porous structure, with pores in the range of mesopores as well as macropores. The NR xerogels have mesopores with an average size of $35 \mathrm{~nm}$ and macropores with diameters ranging from 50 to $210 \mathrm{~nm}$. In the NC xerogels, the average mesopore sizes are $39 \mathrm{~nm}$, while the diameter of macropores ranges from 50 to $550 \mathrm{~nm}$. The xerogel of the magnetic NSs also possesses mesopores ( $35 \mathrm{~nm}$ in average) as well as macropores with sizes up to $460 \mathrm{~nm}$. The porosity in all three samples varies in the same range of pore sizes, which also confirms the versatility of the gel preparation process in terms of achieving similar pore size distributions. Regarding the connection between the individual nanoparticles, the gels were further analyzed by TEM (Figure 3J-L). It can be seen that the single nanoparticles are not in direct contact, but are rather separated by the polymer shell, acting as a spatial spacer. This morphological difference to the previously synthesized nanoparticle gels, ${ }^{4,15}$ in which the inorganic nanoparticles were directly in contact, results in the reduced particle-particle interactions. It therefore explains (i) why the plasmonic properties of the cubic particles strongly resemble those of their building blocks, (ii) why we do not observe strongly increased lifetimes for the NR aerogels as reported previously but rather lifetimes resembling those of the building blocks, and (iii) also that the superparamagnetic particle properties are largely retained in the resulting gels, as will be discussed below. These findings suggest that when macroscopic, homogeneous, and porous gels with nanoscopic properties of the building blocks are desired, the polymer coating is the route of choice. Importantly, concerning the nanoparticle sizes and different shapes, no differences could be observed in comparison to the free polymer-coated nanoparticles, further proving the versatility of the method.

Aerogels. In comparison to the ambient-dried hydrogels, significantly increased porosity can be observed for the aerogels (Figure 4). For all systems, the preparation of monolithic aerogels was successful (Figure S2). Argon physisorption measurements confirm specific surface areas typical for nanocrystal aerogels, in the range of $46 \pm 5,14 \pm 2$, and $38 \pm 4 \mathrm{~m}^{2} / \mathrm{g}$ for semiconductor NR, IONs, and $\mathrm{Au}_{3} \mathrm{Cu}$ NCs, respectively (for the isotherms, see Figure S3). These differences can be attributed to the (i) anisotropy of the NRs and (ii) the significantly differing densities of the core materials. Nevertheless, the obtained specific surface areas are in agreement with the ones in our previous gel network consisting of CdSe/CdS NRs ${ }^{10,44}$ and Pt NCs. ${ }^{8}$ The aerogels were found to be ultralightweight with densities of 0.04, 0.01, and $0.18 \mathrm{~g} / \mathrm{cm}^{3}$ for NRs, IONs, and NCs, respectively. These densities correspond to $0.8 \%, 0.3 \%$, and $1.1 \%$ of the bulk material density $\left(4.82 \mathrm{~g} / \mathrm{cm}^{3}\right.$ for CdS, $5.24 \mathrm{~g} / \mathrm{cm}^{3}$ for $\mathrm{Fe}_{3} \mathrm{O}_{4}$, and $15.46 \mathrm{~g} / \mathrm{cm}^{3}$ for $\mathrm{Au}_{3} \mathrm{Cu}$ ). The highly porous structure can 
be preserved upon aerogelation, which is clearly visible in the SEM images (Figure 3). For all samples, the pore size distribution shows similarities: the aerogels contain mesopores (in the diameter range of $22-50 \mathrm{~nm}$ ) as well as macropores up to $500-600 \mathrm{~nm}$. The average size of the macropores is $154 \mathrm{~nm}$ for the NR gel, $127 \mathrm{~nm}$ for the NS gel, and $110 \mathrm{~nm}$ for the NC gel (see the insets of Figure $4 \mathrm{~A}-\mathrm{C}$ for the distributions). Although the porous structure stays intact, there are structural changes in the network and the connection of the nanoparticles within. From the TEM images, it can be seen that the distance between the single nanoparticles is decreased in comparison to the hydrogels, meaning that the forces during the solvent exchange from water to acetone and the subsequent supercritical drying (i.e., high pressure treatment) influence the interparticle interactions and the densification of the nanoparticle network. However, the particles remain in a short distance to one another. This means, also in case of the aerogels, no direct connection between the particles can be seen. The polymer shell around each nanoparticle still functions as a spacer, and the particles are rather embedded in a quasi-continuous polymer matrix. While the morphology and average particle size show no significant changes compared to the earlier stages, interestingly enough, the NRs in the aerogels are arranged mostly tip-by-tip, which should be caused during the assembly due to electrostatic repulsion. This can be derived from PLQY and lifetime measurements (Figure 5).
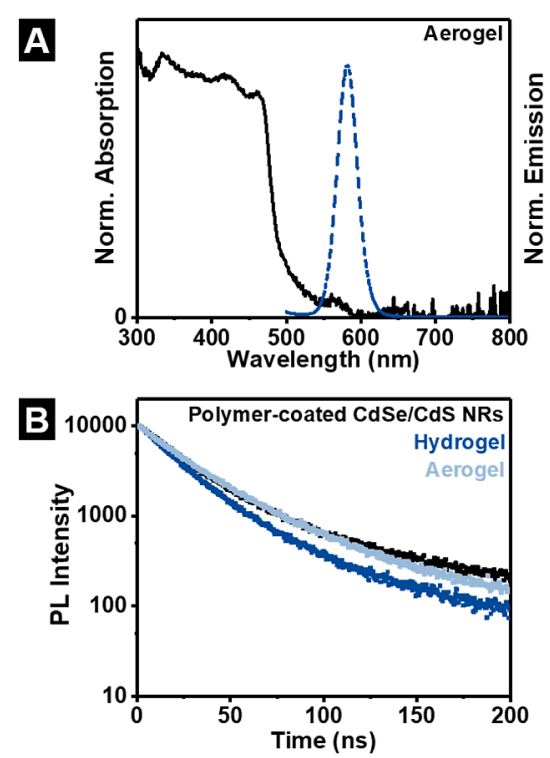

Figure 5. (A) Normalized absorption and PL spectrum of the polymer-coated CdSe/CdS aerogel. (B) PL lifetime decays of the hydro- and aerogels as well as the polymer-coated NR building blocks.

The QY decreases further down to $17.5 \%$ (in the hydrogel, it was at $25.8 \%$ ), while the PL lifetime with a value of $31.0 \mathrm{~ns}$ in the aerogels is similar to the measured value for the free polymer-coated NRs (30.4 ns). This indicates that the NR network consists of electronically mostly decoupled building blocks, and thus, a thin polymer layer separates the particles after aerogelation as well. This is different to the properties of aerogels from similar but nonpolymer-coated building blocks in previous works of our group. ${ }^{4,15}$ In the work mentioned, a particle-to-particle contact between the NRs was observed, which went along with ultralong exciton lifetimes, which we attributed to a delocalization of the excited electrons in the conduction band over more than one $\mathrm{NR}^{10,45}$

The magnetic NSs as well as the subsequently prepared hydrogels and aerogels can be attracted by a nearby magnet during all synthetic steps (Figure S1). Therefore, it could be assumed that the superparamagnetic behavior is preserved in the superstructures. We further analyzed the samples via DC magnetometry to fully characterize their magnetic properties.

The existence of different magnetic phases can induce an exchange bias in the hysteresis cycles. ${ }^{46}$ For measuring the hysteresis cycles under ZFC procedure, the sample is cooled down without applied field. The magnetization at zero field of the hysteresis cycle curve represents the spontaneous ordering of the magnetic moments at low temperature. However, under FC procedure, the sample is cooled down under an applied field $\left(H_{\text {cool }}=0,5,10\right.$, and $\left.30 \mathrm{kOe}\right)$ that causes the onset of exchange interactions between two magnetic phases. These magnetic interactions are determined by the external field applied during cooling down and give place to an asymmetrical shape of the hysteresis cycles, with the left coercive field $\left(H_{c}^{1}\right)$ higher than the right one $\left(H_{c}{ }^{r}\right)$. The average of these two coercive fields is the exchange bias field, $H_{\mathrm{E}}=\left(H_{\mathrm{c}}{ }^{\mathrm{r}}+H_{\mathrm{c}}{ }^{1}\right) / 2$. ZFC hysteresis cycles of IONs at $5 \mathrm{~K}$ show a saturation magnetization $M_{\mathrm{s}}=11.5 \mathrm{emu} / \mathrm{g}$, coercive field $H_{\mathrm{c}}=1.9 \mathrm{kOe}$, and exchange bias $H_{\mathrm{E}}=100 \mathrm{Oe}$, whereas at $300 \mathrm{~K}$, the behavior becomes superparamagnetic and $M_{s}$ remains constant. The presence of $H_{\mathrm{E}}$ is a fingerprint of the coexistence of two magnetic phases, which is besides confirmed by measuring hysteresis cycles at different $H_{\text {cool }}$ (FC procedure). As $H_{\text {cool }}$ increases, $H_{c}$ becomes higher and shifts to more negative fields, as shown in Figure 6A. The high $H_{\mathrm{E}}$ value suggests a strong coupling between the two magnetic phases. A possible explanation of the presence of the magnetic phases is a core of $\mathrm{Fe}_{x} \mathrm{O}$ and a shell of $\mathrm{Fe}_{3} \mathrm{O}_{4}{ }^{47}$

Thermal dependence of the magnetization under ZFC-FC (Figure $6 \mathrm{~B}$ ) shows that the Neel temperature $\left(T_{N}\right)$ of the $\mathrm{Fe}_{x} \mathrm{O}$ core is reached at $190 \mathrm{~K}$, determined from the first derivative of the ZFC curve. The $\mathrm{Fe}_{3} \mathrm{O}_{4}$ phase with better crystallinity could be created in the core/shell form rather than in the spherical form of individual $\mathrm{Fe}_{3} \mathrm{O}_{4}$ nanoparticles, as the core is protected. Above $T_{\mathrm{N}}$, the contribution to the magnetization results mainly from the $\mathrm{Fe}_{3} \mathrm{O}_{4}$ shell. Figure $6 \mathrm{~B}$ shows an increasing magnetization with temperature and peaks at $T=$ $180 \mathrm{~K}$, followed by a decrease due to the thermal fluctuations. Above $180 \mathrm{~K}$, the behavior is superparamagnetic.

The hysteresis curves for the IONs in chloroform, the polymer-coated IONs, and aerogel monoliths of the IONs show similar behavior (Figure 6C,D). The saturation magnetization at 5 and $300 \mathrm{~K}$ remains almost the same for the three samples. The polymer-coated IONs show a higher susceptibility, but it decreases again after gelation. Consequently, the magnetic properties of the IONs are affected neither by the polymer coating nor by the gelation process. Therefore, it can be concluded that by employing the polymer-coating aerogelation route presented in this article, macroscopic monoliths can be achieved, exhibiting the nanoscopic superparamagnetic properties of their building blocks.

\section{CONCLUSIONS}

We have developed a universally applicable method for the synthesis of nanoparticle-based superstructures. Three types of nanoparticles, each of them presented as colloid in a nonpolar solvent, with different characteristics concerning their shape 

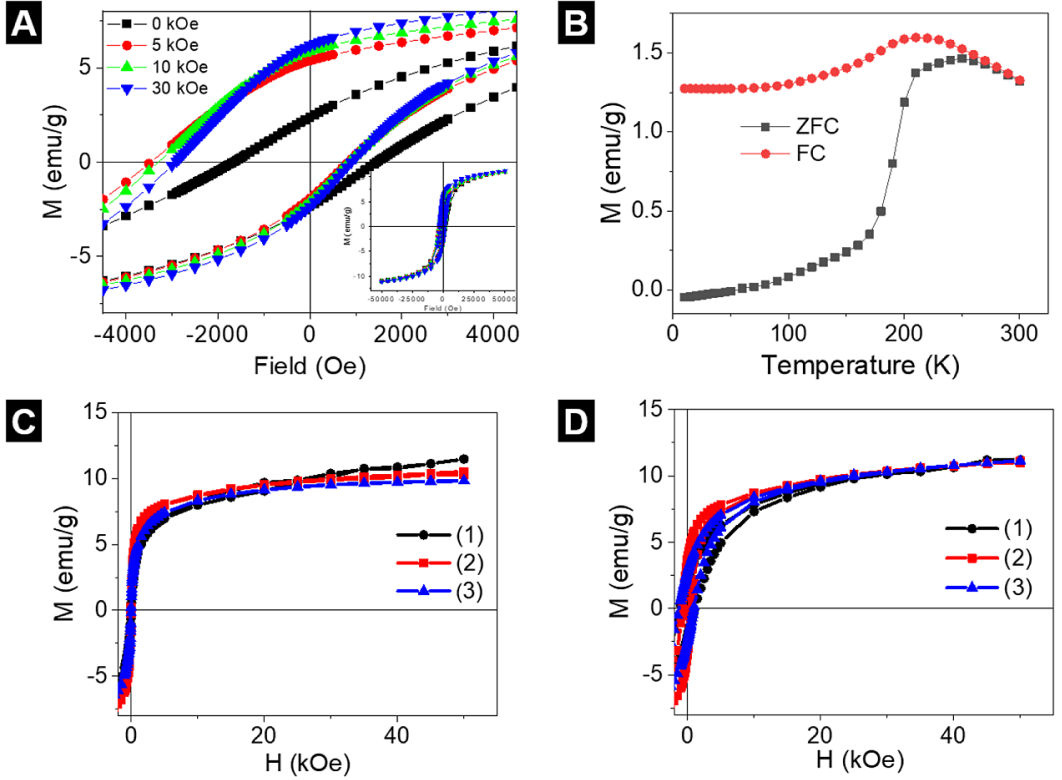

Figure 6. Magnetic characterization. (A) ZFC and FC hysteresis cycles at $5 \mathrm{~K}$ of the uncoated IONs. The shift of $H_{\mathrm{c}}$ to negative fields is a fingerprint of magnetic phase coexistence. The inset shows the hysteresis cycles up to $5 \mathrm{~T}$. (B) ZFC-FC of the uncoated IONs at 100 Oe. Hysteresis cycles at (C) $300 \mathrm{~K}$ and (D) $5 \mathrm{~K}$ of the IONs (1), polymer-coated IONs (2), and aerogels monoliths of the IONs (3).

(rods, spheres, and cubes), their size $(5-40 \mathrm{~nm})$, and their physical properties (photoluminescence, magnetism, and plasmon resonance) have been successfully tested to confirm the versatile character of the polymer-coating procedure, in which the synthesized nanoparticles are encapsulated inside a polymer shell. The destabilization with $\mathrm{Ca}^{2+}$ ions led to the formation of highly porous hydrogels, which could be transformed into highly porous aerogels by supercritical drying. Regarding the physical properties of the monolithic aerogels, no significant changes could be observed between the nanoparticle superstructures in comparison to the solutions of the nanoparticles, proving the noninvasive character of the employed method. Therefore, the suggested procedure of gelating via polymer-coated nanoparticles is a highly versatile route for the elaboration of macroscopic superstructures. Since the resulting materials exhibit the nanoscopic properties and seem to consist of largely electronically decoupled building blocks, this route especially becomes relevant when selfsupported macroscale monoliths are needed, exhibiting the nanoscopic properties of their tailored nanoparticle building blocks. Due to the open pore structure, the high porosity, and the preserved nanoscopic properties, the presented method allows the preparation of novel biosensing platforms in the future, where the properties of the building blocks (fluorescent, plasmonic, and magnetic) can be merged with the widely functionalizable polymer coating, offering the detection of gaseous analytes as well.

\section{ASSOCIATED CONTENT}

\section{SI Supporting Information}

The Supporting Information is available free of charge at https://pubs.acs.org/doi/10.1021/acsanm.1c00636.

Additional photographs of the hydrogels and aerogels as well as the isotherms of the argon physisorption measurements (PDF)

\section{AUTHOR INFORMATION}

\section{Corresponding Author}

Nadja C. Bigall - Institute of Physical Chemistry and Electrochemistry, Leibniz Universität Hannover, Hanover 30167, Germany; Cluster of Excellence PhoenixD (Photonics, Optics, and Engineering - Innovation Across Disciplines), Hanover 30167, Germany; 이이.org/0000-0003-01711106; Email: nadja.bigall@pci.uni-hannover.de

\section{Authors}

Laura Altenschmidt - Institute of Physical Chemistry and Electrochemistry, Leibniz Universität Hannover, Hanover 30167, Germany

Sara Sánchez-Paradinas - Institute of Physical Chemistry and Electrochemistry, Leibniz Universität Hannover, Hanover 30167, Germany

Franziska Lübkemann - Institute of Physical Chemistry and Electrochemistry, Leibniz Universität Hannover, Hanover 30167, Germany; (1) orcid.org/0000-0003-4783-0223

Dániel Zámbó - Institute of Physical Chemistry and Electrochemistry, Leibniz Universität Hannover, Hanover 30167, Germany; 이이.org/0000-0001-7671-039X

Abuelmagd M. Abdelmonem - Institute of Physical Chemistry and Electrochemistry, Leibniz Universität Hannover, Hanover 30167, Germany; Food Technology Research Institute, Agricultural Research Center, Giza 12619, Egypt

Henrik Bradtmüller - Institute of Physical Chemistry and Electrochemistry, Leibniz Universität Hannover, Hanover 30167, Germany; Institute of Physical Chemistry, Westfälische Wilhelms-Universität Münster, Münster D48149, Germany

Atif Masood - Fachbereich Physik and WZMW, Philipps Universität Marburg, Marburg 35032, Germany

Irene Morales - Instituto de Magnetismo Aplicado, UCMADIF-CSIC, Las Rozas 28230, Spain

Patricia de la Presa - Instituto de Magnetismo Aplicado, UCM-ADIF-CSIC, Las Rozas 28230, Spain 
Alexander Knebel - Institute of Physical Chemistry and Electrochemistry, Leibniz Universität Hannover, Hanover 30167, Germany; Institute of Functional Interfaces (IFG), Karlsruhe Institute of Technology, Eggenstein-Leopoldshafen 76344, Germany; ○ orcid.org/0000-0002-5866-1106

Miguel Angel García García-Tuñón - Instituto de Cerámica y Consejo Vidrio, CSIC \& IMDEA Nanociencia, Madrid E28049, Spain

Beatriz Pelaz - Centro Singular de Investigación en Química Biolóxica e Materiais Moleculares (CiQUS), Departamento de Química Inorgánica, Universidade de Santiago de Compostela, Santiago de Compostela 15782, Spain; (1) orcid.org/0000-0002-4626-4576

Karen D. J. Hindricks - Institute of Inorganic Chemistry, Leibniz Universität Hannover, Hanover 30167, Germany; Cluster of Excellence PhoenixD (Photonics, Optics, and Engineering - Innovation Across Disciplines), Hanover 30167, Germany

Peter Behrens - Institute of Inorganic Chemistry, Leibniz Universität Hannover, Hanover 30167, Germany; Cluster of Excellence Hearing4all, Hanover 30167, Germany; Cluster of Excellence PhoenixD (Photonics, Optics, and Engineering Innovation Across Disciplines), Hanover 30167, Germany

Wolfgang J. Parak - Fachbereich Physik und Chemie, CHyN, Universität Hamburg, Hamburg 22607, Germany;

(1) orcid.org/0000-0003-1672-6650

Complete contact information is available at:

https://pubs.acs.org/10.1021/acsanm.1c00636

\section{Author Contributions}

${ }^{\#}$ L.A. and S. S.-P. contributed equally to this work. The manuscript was written through contributions of all authors. All authors have given approval to the final version of the manuscript.

Notes

The authors declare no competing financial interest.

\section{ACKNOWLEDGMENTS}

The authors (L. A., S. S.-P., H. B., F. L., D. Z., and N.C.B.) are grateful for the financial support from the German Federal Ministry of Education and Research (BMBF) within the framework of the program NanoMatFutur (support code 03X5525). Furthermore, the project leading to these results has in part received funding from the European Research Council (ERC) under the European Union's Horizon 2020 research and innovation program (grant agreement no. 714429). The project has in parts been funded by the Deutsche Forschungsgemeinschaft (DFG, German Research Foundation) under Germany's Excellence Strategy within the Cluster of Excellence PhoenixD (EXC 2122, project ID: 390833453), the Cluster of Excellence AIM (EXC 2056, project ID: 390715994), and the Cluster of Excellence Hearing4all (EXC 1077/1). B.P. thanks Spanish MINECOAEI/FEDER (no. PID2019-111218RB-I00), the RyC program (grant agreement no. 2017-23457), and the Xunta de Galicia (Centro singular de investigación de Galicia accreditation 2019-2022, no. ED431G 2019/03). The authors thank Prof. Caro and Prof. Feldhoff for access to a scanning electron microscope, Lars Klepzig for help with the nitrogen adsorption measurements, and the Laboratorium of Nano- and Quantum Engineering of the Leibniz Universität Hannover for the support. Furthermore, they would like to thank PD Dr.
Carsten Zeilinger for the provision of the ultracentrifuges at the Centre of Biomolecular Drug Research (BMWZ) in Hannover.

\section{REFERENCES}

(1) Mohanan, J. L.; Brock, S. L. A New Addition to the Aerogel Community: Unsupported CdS Aerogels with Tunable Optical Properties. J. Non-Cryst. Solids 2004, 350, 1-8.

(2) Mohanan, J. L.; Arachchige, I. U.; Brock, S. L. Porous Semiconductor Chalcogenide Aerogels. Science 2005, 37, 397-400.

(3) Bigall, N. C.; Herrmann, A.-K.; Vogel, M.; Rose, M.; Simon, P.; Carrillo-Cabrera, W.; Dorfs, D.; Kaskel, S.; Gaponik, N.; Eychmüller, A. Hydrogels and Aerogels from Noble Metal Nanoparticles. Angew. Chem., Int. Ed. 2009, 48, 9731-9734.

(4) Sánchez-Paradinas, S.; Dorfs, D.; Friebe, S.; Freytag, A.; Wolf, A.; Bigall, N. C. Aerogels from CdSe/CdS Nanorods with Ultra-Long Exciton Lifetimes and High Fluorescence Quantum Yields. Adv. Mater. 2015, 27, 6152-6156.

(5) Rusch, P.; Zámbó, D.; Bigall, N. C. Control over Structure and Properties in Nanocrystal Aerogels at the Nano-, Micro-, and Macroscale. Acc. Chem. Res. 2020, 53, 2414-2424.

(6) Ziegler, C.; Wolf, A.; Liu, W.; Herrmann, A.-K.; Gaponik, N.; Eychmüller, A. Modern Inorganic Aerogels. Angew. Chem., Int. Ed. 2017, 56, 13200-13221.

(7) Sayevich, V.; Cai, B.; Benad, A.; Haubold, D.; Sonntag, L.; Gaponik, N.; Lesnyak, V.; Eychmüller, A. 3D Assembly of AllInorganic Colloidal Nanocrystals into Gels and Aerogels. Angew. Chem., Int. Ed. 2016, 55, 6334-6338.

(8) Naskar, S.; Freytag, A.; Deutsch, J.; Wendt, N.; Behrens, P.; Köckritz, A.; Bigall, N. C. Porous Aerogels from Shape-Controlled Metal Nanoparticles Directly from Nonpolar Colloidal Solution. Chem. Mater. 2017, 29, 9208-9217.

(9) Freytag, A.; Sánchez-Paradinas, S.; Naskar, S.; Wendt, N.; Colombo, M.; Pugliese, G.; Poppe, J.; Demirci, C.; Kretschmer, I.; Bahnemann, D. W.; Behrens, P.; Bigall, N. C. Versatile Aerogel Fabrication by Freezing and Subsequent Freeze-Drying of Colloidal Nanoparticle Solutions. Angew. Chem., Int. Ed. 2016, 55, 1200-1203.

(10) Rusch, P.; Niemeyer, F.; Pluta, D.; Schremmer, B.; Lübkemann, F.; Rosebrock, M.; Schäfer, M.; Jahns, M.; Behrens, P.; Bigall, N. C. Versatile Route to Core-Shell Reinforced Network Nanostructures. Nanoscale 2019, 11, 15270-15278.

(11) Li, Y.; Liu, Q.; Hess, A. J.; Mi, S.; Liu, X.; Chen, Z.; Xie, Y.; Smalyukh, I. I. Programmable Ultralight Magnets via Orientational Arrangement of Ferromagnetic Nanoparticles within Aerogel Hosts. ACS Nano 2019, 13, 13875-13883.

(12) Liu, Q.; Mundoor, H.; Sheetah, G. H.; Smalyukh, I. I. Plasmonic Gold-Cellulose Nanofiber Aerogels. Opt. Express 2020, 28, No. 34237.

(13) Janulevicius, M.; Klimkevičius, V.; Mikoliunaite, L.; Vengalis, B.; Vargalis, R.; Sakirzanovas, S.; Plausinaitiene, V.; Zilinskas, A.; Katelnikovas, A. Ultralight Magnetic Nanofibrous GdPO ${ }_{4}$ Aerogel. ACS Omega 2020, 5, 14180-14185.

(14) Lesnyak, V.; Voitekhovich, S. V.; Gaponik, P. N.; Gaponik, N.; Eychmüller, A. CdTe Nanocrystals Capped with a Tetrazolyl Analogue of Thioglycolic Acid: Aqueous Synthesis, Characterization, and Metal-Assisted Assembly. ACS Nano 2010, 4, 4090-4096.

(15) Zámbó, D.; Schlosser, A.; Rusch, P.; Lübkemann, F.; Koch, J.; Pfnür, H.; Bigall, N. C. A Versatile Route to Assemble Semiconductor Nanoparticles into Functional Aerogels by Means of Trivalent Cations. Small 2020, 16, No. 1906934.

(16) Müller, D.; Zámbó, D.; Dorfs, D.; Bigall, N. C. Cryoaerogels and Cryohydrogels as Efficient Electrocatalysts. Small 2021, 17, No. 2007908.

(17) Müller, D.; Klepzig, L. F.; Schlosser, A.; Dorfs, D.; Bigall, N. C. Structural Diversity in Cryoaerogel Synthesis. Langmuir 2021, 37, 5109-5117.

(18) Schlosser, A.; Meyer, L. C.; Lübkemann, F.; Miethe, J. F.; Bigall, N. C. Nanoplatelet Cryoaerogels with Potential Application in 
Photoelectrochemical Sensing. Phys. Chem. Chem. Phys. 2019, 21, 9002-9012.

(19) Bigall, N. C.; Parak, W. J.; Dorfs, D. Fluorescent, Magnetic and Plasmonic-Hybrid Multifunctional Colloidal Nano Objects. Nano Today 2012, 7, 282-296.

(20) Giner-Casares, J. J.; Henriksen-Lacey, M.; Coronado-Puchau, M.; Liz-Marzán, L. M. Inorganic Nanoparticles for Biomedicine: Where Materials Scientists Meet Medical Research. Mater. Today 2016, 19, 19-28.

(21) O’Neal, D. P.; Hirsch, L. R.; Halas, N. J.; Payne, J. D.; West, J. L. Photo-Thermal Tumor Ablation in Mice Using near InfraredAbsorbing Nanoparticles. Cancer Lett. 2004, 209, 171-176.

(22) Corr, S. A.; Byrne, S. J.; Tekoriute, R.; Meledandri, C. J.; Brougham, D. F.; Lynch, M.; Kerskens, C.; O’Dwyer, L.; Gun'ko, Y. K. Linear Assemblies of Magnetic Nanoparticles as MRI Contrast Agents. J. Am. Chem. Soc. 2008, 130, 4214-4215.

(23) Walling, M.; Novak, J.; Shepard, J. R. E. Quantum Dots for Live Cell and In Vivo Imaging. IJMS 2009, 10, 441-491.

(24) Jimenez De Aberasturi, D.; Montenegro, J.-M.; Ruiz De Larramendi, I.; Rojo, T.; Klar, T. A.; Alvarez-Puebla, R.; Liz-Marzán, L. M.; Parak, W. J. Optical Sensing of Small Ions with Colloidal Nanoparticles. Chem. Mater. 2012, 24, 738-745.

(25) Soliman, M. G.; Pelaz, B.; Parak, W. J.; del Pino, P. Phase Transfer and Polymer Coating Methods toward Improving the Stability of Metallic Nanoparticles for Biological Applications. Chem. Mater. 2015, 27, 990-997.

(26) Kodanek, T.; Banbela, H. M.; Naskar, S.; Adel, P.; Bigall, N. C.; Dorfs, D. Phase Transfer of 1- and 2-Dimensional Cd-Based Nanocrystals. Nanoscale 2015, 7, 19300-19309.

(27) Zhang, F.; Lees, E.; Amin, F.; Rivera, P.; Yang, F.; Mulvaney, P.; Parak, W. J. Polymer-Coated Nanoparticles: A Universal Tool for Biolabelling Experiments. Small 2011, 7, 3113-3127.

(28) Pellegrino, T.; Manna, L.; Kudera, S.; Liedl, T.; Koktysh, D.; Rogach, A. L.; Keller, S.; Rädler, J.; Natile, G.; Parak, W. J. Hydrophobic Nanocrystals Coated with an Amphiphilic Polymer Shell: A General Route to Water Soluble Nanocrystals. Nano Lett. 2004, 4, 703-707.

(29) Sperling, R. A.; Parak, W. J. Surface Modification, Functionalization and Bioconjugation of Colloidal Inorganic Nanoparticles. Philos. Trans. R. Soc., A 2010, 368, 1333-1383.

(30) Corato, R. D.; Quarta, A.; Piacenza, P.; Ragusa, A.; Figuerola, A.; Buonsanti, R.; Cingolani, R.; Manna, L.; Pellegrino, T. Water Solubilization of Hydrophobic Nanocrystals by Means of Poly(Maleic Anhydride-Alt-1-Octadecene). J. Mater. Chem. 2008, 18, 1991-1996.

(31) Jańczewski, D.; Tomczak, N.; Han, M.-Y.; Vancso, G. J. Synthesis of Functionalized Amphiphilic Polymers for Coating Quantum Dots. Nat. Protoc. 2011, 6, 1546-1553.

(32) Yakovlev, A. V.; Zhang, F.; Zulqurnain, A.; Azhar-Zahoor, A.; Luccardini, C.; Gaillard, S.; Mallet, J.-M.; Tauc, P.; Brochon, J.-C.; Parak, W. J.; Feltz, A.; Oheim, M. Wrapping Nanocrystals with an Amphiphilic Polymer Preloaded with Fixed Amounts of Fluorophore Generates FRET-Based Nanoprobes with a Controlled Donor/ Acceptor Ratio. Langmuir 2009, 25, 3232-3239.

(33) Polo, E.; del Pino, P.; Pelaz, B.; Grazu, V.; De La Fuente, J. M. Plasmonic-Driven Thermal Sensing: Ultralow Detection of Cancer Markers. Chem. Commun. 2013, 49, 3676-3678.

(34) Huang, H. T.; Garu, P.; Li, C. H.; Chang, W. C.; Chen, B. W.; Sung, S. Y.; Lee, C. M.; Chen, J. Y.; Hsieh, T. F.; Sheu, W. J.; Ouyang, H.; Wang, W. C.; Chang, C. R.; Wang, C. L.; Hsu, M. S.; Wei, Z. H. Magnetoresistive Biosensors for Direct Detection of Magnetic Nanoparticle Conjugated Biomarkers on a Chip. SPIN 2019, 09, No. 1940002.

(35) Li, Y.; Wang, N.; Huang, X.; Li, F.; Davis, T. P.; Qiao, R.; Ling, D. Polymer-Assisted Magnetic Nanoparticle Assemblies for Biomedical Applications. ACS Appl. Bio Mater. 2020, 3, 121-142.

(36) Lin, C.-A. J.; Sperling, R. A.; Li, J. K.; Yang, T.-Y.; Li, P.-Y.; Zanella, M.; Chang, H.; Parak, W. J. Design of an Amphiphilic Polymer for Nanoparticle Coating and Functionalization. Small 2008, 4, 334-341.
(37) Carbone, L.; Nobile, C.; De Giorgi, M.; Sala, F. D.; Morello, G.; Pompa, P.; Hytch, M.; Snoeck, E.; Fiore, A.; Franchini, I. R.; Nadasan, M.; Silvestre, A. F.; Chiodo, L.; Kudera, S.; Cingolani, R.; Krahne, R.; Manna, L. Synthesis and Micrometer-Scale Assembly of Colloidal CdSe/CdS Nanorods Prepared by a Seeded Growth Approach. Nano Lett. 2007, 7, 2942-2950.

(38) Yu, W. W.; Qu, L.; Guo, W.; Peng, X. Experimental Determination of the Extinction Coefficient of CdTe, CdSe, and CdS Nanocrystals. Chem. Mater. 2003, 15, 2854-2860.

(39) Yu, W. W.; Falkner, J. C.; Yavuz, C. T.; Colvin, V. L. Synthesis of Monodisperse Iron Oxide Nanocrystals by Thermal Decomposition of Iron Carboxylate Salts. Chem. Commun. 2004, 2306-2307.

(40) Liu, Y.; Walker, A. R. H. Monodisperse Gold-Copper Bimetallic Nanocubes: Facile One-Step Synthesis with Controllable Size and Composition. Angew. Chem., Int. Ed. 2010, 49, 6781-6785.

(41) Del pino, P.; Yang, F.; Pelaz, B.; Zhang, Q.; Kantner, K.; Hartmann, R.; Martinez de baroja, N.; Gallego, M.; Möller, M.; Manshian, B. B.; Soenen, S. J.; Riedel, R.; Hampp, N.; Parak, W. J. Basic Physicochemical Properties of Polyethylene Glycol Coated Gold Nanoparticles That Determine Their Interaction with Cells. Angew. Chem., Int. Ed. 2016, 55, 5483-5487.

(42) Hühn, J.; Carrillo-Carrion, C.; Soliman, M. G.; Pfeiffer, C.; Valdeperez, D.; Masood, A.; Chakraborty, I.; Zhu, L.; Gallego, M.; Yue, Z.; Carril, M.; Feliu, N.; Escudero, A.; Alkilany, A. M.; Pelaz, B.; del Pino, P.; Parak, W. J. Selected Standard Protocols for the Synthesis, Phase Transfer, and Characterization of Inorganic Colloidal Nanoparticles. Chem. Mater. 2017, 29, 399-461.

(43) Wen, D.; Herrmann, A.-K.; Borchardt, L.; Simon, F.; Liu, W.; Kaskel, S.; Eychmüller, A. Controlling the Growth of Palladium Aerogels with High-Performance toward Bioelectrocatalytic Oxidation of Glucose. J. Am. Chem. Soc. 2014, 136, 2727-2730.

(44) Lübkemann, F.; Rusch, P.; Getschmann, S.; Schremmer, B.; Schäfer, M.; Schulz, M.; Hoppe, B.; Behrens, P.; Bigall, N. C.; Dorfs, D. Reversible Cation Exchange on Macroscopic CdSe/CdS and CdS Nanorod Based Gel Networks. Nanoscale 2020, 12, 5038-5047.

(45) Rusch, P.; Schremmer, B.; Strelow, C.; Mews, A.; Dorfs, D.; Bigall, N. C. Nanocrystal Aerogels with Coupled or Decoupled Building Blocks. J. Phys. Chem. Lett. 2019, 10, 7804-7810.

(46) Nogués, J.; Sort, J.; Langlais, V.; Skumryev, V.; Suriñach, S.; Muñoz, J. S.; Baró, M. D. Exchange Bias in Nanostructures. Phys. Rep. 2005, 422, 65-117.

(47) Redl, F. X.; Black, C. T.; Papaefthymiou, G. C.; Sandstrom, R. L.; Yin, M.; Zeng, H.; Murray, C. B.; O’Brien, S. P. Magnetic, Electronic, and Structural Characterization of Nonstoichiometric Iron Oxides at the Nanoscale. J. Am. Chem. Soc. 2004, 126, 14583-14599. 